\title{
Wie Hassenstein und Ravenstein Opfer des Gletscherrückgangs wurden
}

\author{
Wolfgang $\mathrm{Crom}^{1}$ (1)
}

Received: 26 November 2020 / Accepted: 7 January 2021 / Published online: 3 March 2021

(c) The Author(s) 2021

\section{Zusammenfassung}

Cartographers are rarely honoured by the use of their name as a toponym (proper name of a topographic object). On Kilimanjaro, however, the first climber and geographer, Hans Meyer, commemorated two outstanding contemporaries known in cartography, Ravenstein and Hassenstein. He named lava rocks after them which protruded from their embrace of ice.

\section{Die Namensgebung des Kilimandscharo}

Die Nachricht von der Schneebedeckung der großen und hohen Vulkanmassive im tropischen Afrika hat im wissenschaftlichen Europa Mitte des 19. Jahrhunderts zunächst heftiges Gelächter und beißenden Spott ausgelöst. Den Beschreibungen der in Diensten der britischen Church Missionary Society stehenden Missionare Johannes Rebmann (1820-1876) und Johann Ludwig Krapf (1810-1881) wurde seitens der Royal Geographical Society of London, Division: namentlich durch den Geographen William Desborough Cooley (1795?-1883), kein Glaube geschenkt und ihre Beobachtungen auf Hirngespinste oder Fehldeutungen aufgrund Rebmanns Kurzsichtigkeit zurückgeführt. Selbst die Berichte des Forschungsreisenden Carl von der Decken (1833-1865) und seines Begleiters, des englischen Geologen Richard Thornton (1838-1863), über nächtliche Schneefälle am Kilimandscharo wurden zunächst noch mit Polemik zurückgewiesen (Meyer 1890, S. 7f.). Erst nachdem weitere Forschungsreisende das Gebiet um das Vulkanmassiv bereisten und über seine Eisbedeckung berichteten, änderte sich das Meinungsbild. So hat August Petermann (1822-1878) in seiner 1859 veröffentlichten „Originalkarte zu Burton's und Speke's Entdeckungen in Inner Afrika 1857 u. 1858 " die Schneebedeckung noch nicht berücksichtigt, obwohl die 1850 von Rebmann angefertigte Kartenskizze wenigstens den schriftlichen Vermerk führte: „Kilimandjaro covered with eternal snow". Diesen Weg schlug 1863 auch Heinrich Kiepert (1818-1899) in der nach von der Decken

Wolfgang Crom

wolfgang.crom@sbb.spk-berlin.de

1 Staatsbibliothek zu Berlin, Berlin, Germany gezeichneten Karte ein, indem das Gebirge im Titel „Karte des Schneegebirges Kilima-Ndjaro " bezeichnetet wurde. Ebenso verwendete Bruno Hassenstein (1839-1902) in seinen Karten von 1864 bzw. Hassenstein 1868 (Abb. 1) die Bezeichnung Schneeberg Kilima-Ndscharo, ohne dass eine zeichnerische Entsprechung für das Vorkommen von Schnee oder Eis in den Karten schon zum Einsatz gekommen wäre. Die schriftliche Hervorhebung der Schneebedeckung im Titel oder beim Toponym der frühen Karten des äquatornahen Vulkanmassivs belegt damit die Wirkung dieses Phänomens auf die Gelehrtenwelt eindrucksvoll.

Benennung und Schreibweise sind in der frühen Zeit und in den ersten Karten ebenfalls noch nicht ganz eindeutig. So finden sich Kilima-Njaro, Kilima-Ndjaro oder KilimaNdscharo, was auf den Versuch der Berücksichtigung der Aussprache des von den Bewohnern übernommenen Toponyms hindeutet. Es deutet aber auch auf das Problem unterschiedlicher Quellen und Aussprache zur Benennung des Bergmassivs hin, was sich in den Karten niederschlägt, weshalb diese teilweise mit angegeben werden. So präzisiert Hassenstein in der schon erwähnten Karte von 1864, die nach Reiseberichten verschiedener Autoren erstellt worden war: ,Schneeberg Kilima-Ndscharo der Wateita “, während Rebmann Suaheli als Gewährsleute angeben hatte, die an der Küste um Mombasa, seiner Missionsstation, siedelten. Als er das Gebiet um das Vulkanmassiv später bereiste und den Berg selbst zu sehen bekam, wurde ihm von den dort siedelnden Chagga die Bezeichnung Kibo übermittelt. Ein drittes Toponym gelangte schließlich mit Dónye Ebor in die Karten; es ist der Name, der von den im nördlichen Bereich siedelnden Massai verwendet wurde und ebenfalls in verschiedenen Schreibweisen überliefert ist. Immerhin war bereits erkannt, dass die Gipfelregion geomorphologisch 


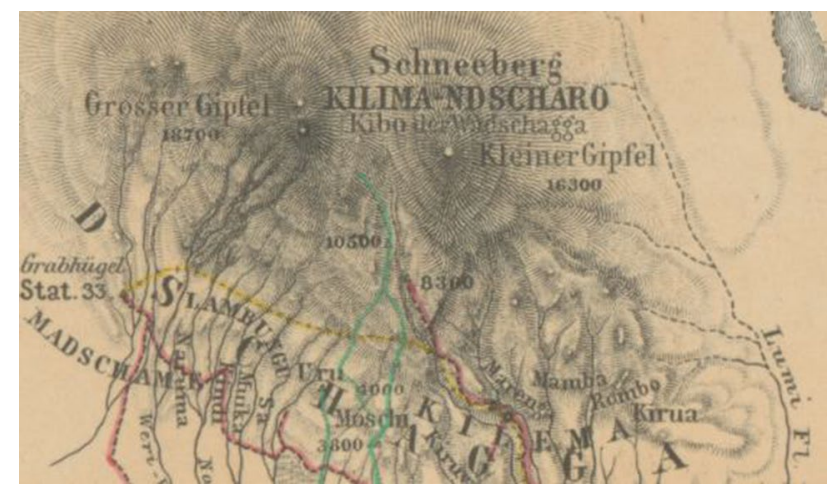

Abb. 1 Ausschnitt aus Hassenstein (1868): Das Gebiet der beiden Reisen des Baron C. C. von der Decken zum Schneeberg KilimaNdscharo in den Jahren 1861 and 1862. 1:1.000.000. Quelle: Staatsbibliothek zu Berlin Kart. GfE J 5, 371

in mehrere Bereiche gegliedert werden konnte. Nachdem zunächst in den Karten Großer und Kleiner Gipfel unterschieden wurde (z.B. Kiepert 1863 oder Hassenstein 1868, der nun die Bezeichnung Kibo der Wadschagga hinzufügt, Abb. 1), setzten sich schließlich Kilimandscharo für das gesamte Vulkanmassiv und Kibo für den vergletscherten Hauptgipfel durch, die weiteren Gipfel bekamen die Benennungen Mawensi und Schira.

\section{Koloniale Toponomastik}

Die Problematik der Benennungen kann als symptomatisch für die imperialistische Kolonialzeit gesehen werden (Stolz und Warnke 2015), was auch zu Klagen seitens der führenden Kartographen des Berliner Kolonialkartographischen Instituts führte: "Besondere Schwierigkeiten ergeben sich auch bei der Beschriftung. Die Reisenden liefern oft für dasselbe Objekt die verschiedenartigsten Namenfassungen. Ursachen dafür sind einmal die größere oder geringere Kenntnis der Landessprachen des Aufnehmers selbst, sodann die Unzuverlässigkeit der Führer, die auch, je nach ihrer Stammesangehörigkeit, häufig für denselben Fluß, Ort usw. ganz verschiedene Namen haben, und endlich Mißverständnisse aller Art", merken Sprigade und Moisel noch 1914 (S. 540) an. Die Kartographen forderten keine exakte sprachwissenschaftliche Analyse, sondern eine pragmatische, aus der Landeskenntnis gewonnene Ableitung und Festlegung, um zu schnellen und verbindlichen Ergebnissen kommen zu können. Den in den Kolonien tätigen Verwaltungen sollte dabei die Aufgabe übertragen werden, die von den Reisenden erfassten geographischen Namen nach einheitlichen Kriterien und nach Möglichkeit unter Ausnutzung der vor Ort erworbenen indigenen Sprachkenntnisse in die deutsche Sprache umzusetzen und eine gültige Schreibweise festzulegen. Diese Forderung fußte auf der bereits 1892 gegründeten Kommission zur Regelung der einheitlichen Schreib- und Sprechweise der geographischen Namen in den deutschen Schutzgebieten, deren Auftrag im 3. Band des Deutschen Kolonialblattes unter den amtlichen Mitteilungen angezeigt wurde (Deutsches Kolonialblatt 1892, S. 407 ff.). In Band 14 derselben Zeitschrift erschienen 1903 die Grundsätze für die Namengebung, Namenübersetzung, Schreib- und Sprechweise der geographischen Namen in den deutschen Schutzgebieten (Deutsches Kolonialblatt 103, S. 453 f.), die insbesondere Endonyme, in der Sprache der ansässigen Bevölkerungsgruppe gebildete Ortsnamen, bevorzugten und die Übertragung der Lautfolge in die deutsche Schreibweise vorschrieben. ${ }^{1}$

Die fortgesetzte Erschließung und die stetig verbesserten Ortskenntnisse erzeugten dabei eine Dynamik, die sich sehr gut an den Karten des Großen Deutschen Kolonialatlas ablesen lässt. Der als Lieferungswerk konzipierte und von 1901 bis 1914 im Auftrag der Kolonialabtheilung des Auswärtigen Amtes bei Reimer erschienene Kartenband belegt dies eindrucksvoll, da manche Kartenblätter in zeitlich kurzen Abständen mehrfach überarbeitet wurden, um den jeweils neuesten Kenntnisstand der geographischen Erkundung kartographisch zu manifestieren. Ein flüchtiger Blick auf ein identisches Kartenblatt verschiedener Jahre (z.B. Karte von Kamerun 1:1.000.000, Blatt 2: Tschad von 1901 und 1909) lässt auffällige Änderungen in der Dichte der dargestellten Landschaftselemente und der ihnen zugewiesenen Toponyme erkennen. Die verschiedenen Bearbeitungszustände weisen neben der Änderung in der Grenzziehung zu benachbarten Schutzgebieten auf eine sehr aktive Phase der Kartierung bzw. der Erhebung der Toponyme hin. Die Änderungen des enthaltenen Namensgutes beziehen sich nicht nur auf die möglicherweise veränderte Lage der bezeichneten Objekte, auf eine andere Schreibweise oder auf ein erwartetes Mehr an Toponymen, sondern sie betreffen auch Neubenennungen oder sogar das Entfernen von Ortsnamen, nachdem eine Überprüfung alter Reiseberichte als bisherige Quelle stattfinden konnte. Dieses Phänomen belegt nicht nur die Problematik der Gewissenhaftigkeit von Gewährsleuten und deren Sprachkenntnisse (Fragende und

\footnotetext{
${ }^{1} \mathrm{Da}$ die indigenen Sprachen bislang keine Verschriftlichung kannten, traf die Kolonialmacht mit dieser Vorgehensweise der verbindlichen Einführung zur Verschriftlichung von Toponymen Festlegungen für eine amtliche Schreibweise, deren Ausgangspunkt auf der eigenen Landessprache basierte. Somit wurden Endonyme in einem allochthonen, also fremd bestimmtem, Schrift- und Lautsystem fixiert.
} 
Befragte), ${ }^{2}$ sondern auch die Akribie der Kartographen in ihren Werkstätten. ${ }^{3}$

Es ist dabei jedoch zu bedenken, dass viele Reisende noch keinen fachlich geschulten Blick auf die mannigfaltigen und neuen Eindrücke haben konnten, sondern viele Phänomene einzig aus der Position des Europäers betrachteten. Es ist beispielsweise fraglich, ob immer richtig zwischen temporärer oder dauerhafter Siedlung unterschieden wurde. Und auch der Name eines Ortes konnte sich ändern, denn in vielen Regionen Afrikas erfolgte die Benennung in der Regel nach dem Oberhaupt. Mit Änderung der Gebietszugehörigkeit (Herrschaftswechsel) oder des Oberhauptes änderte oder verlagerte sich auch der Ortsname (Meyer 1900, S. 102), was bei den vielen europäischen Reisenden zwangsläufig zu Irrtümern führte. Es hatten auch nicht alle geographische Objekte einen Eigennamen, vielmehr wurden Gattungsbegriffe wie -berg oder -bach meist mit dem Landschaftsnamen kombiniert, was zu Verwechslungen führte. Hans Meyer (1858-1929), der sich um die wissenschaftliche Erschließung des Kilimandscharo große Verdienste erworben hatte, beklagte denn auch ein Durcheinander der Ortsund Gewässernamen in den Karten: „Ist die Planlosigkeit in der Namengebung bisher schon groß gewesen, so steigert sich die Verwirrung noch dadurch, daß thatsächlich ein und derselbe Bach oder Fluß in den verschiedenen Abschnitten seines Laufes von demselben Volksstamm verschieden benannt wird" (Meyer 1900, S. 101). Deshalb bevorzugte er in seinen Karten die Verwendung von Endonymen der jeweiligen Gruppe in ihrem Territorium.

\section{Neue Toponyme am Kilimandscharo}

Andererseits hatte gerade Meyer am Kilimandscharo zahlreiche Exonyme, d.h. Ortsnamen in einer anderen als der von der ansässigen Bevölkerungsgruppe verwendeten Sprache, gebildet und in seinen Karten manifestiert. Dabei handelte es sich aber nicht etwa um Übersetzungen oder Parallelnamen, sondern um Neubenennungen für bislang unbenannte Landschaftselemente in nicht dauerhaft besiedelten oder bewirtschafteten Regionen der Hochlagen des Kilimandscharo. Bereits während seiner am 6. Oktober 1889

\footnotetext{
${ }^{2}$ Der Geograph Alfred Hettner resümiert: „,...) die Kenntnis der Schneeberge und der großen Seen in Ost-Afrika ist zuerst nicht durch unmittelbare Beobachtung, sondern durch Mitteilung der Eingeborenen gewonnen worden. Die Karte kann sich so auch abseits vom eigenen Reiseweg füllen. Die Bedeutung mancher Reisenden hat hauptsächlich in der Kunst des Fragens bestanden“ (Hettner 1927, S. 173)

${ }^{3}$ Zur Geschichte der Kartographie Ostafrikas und des Kilimandscharo s. Brunner 1989 und 2004, Crom 2019, Demhardt 2004, Pillewizer 1941
}

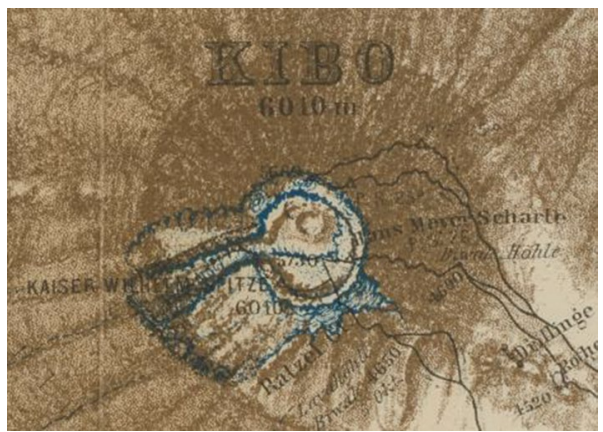

Abb. 2 Ausschnitt aus Meyer/Hassenstein Originalkarte des KilimaNdscharo. 1:250.000. Beilage zu Meyer 1890. Quelle: Staatsbibliothek zu Berlin Kart. GfE J 6832

erfolgten Erstbesteigung des Kibo-Gipfels benannte er den im östlichen Gipfelbereich überquerten Gletscher RatzelGletscher (Abb. 2) und ehrte damit den Leipziger Geographen Friedrich Ratzel (1844-1904). Den höchsten Punkt des Kilimandscharo und damit Afrikas und des Deutschen Kaiserreichs benannte er standesgemäß nach dem höchsten Repräsentanten Kaiser-Wilhelm-Spitze (Wilhelm II. von Preußen, 1859-1941). Im Zuge seiner 1898 durchgeführten Expedition untersuchte er insbesondere die auf der Westund Südseite befindlichen Gletscherfelder und konnte dabei erstmals eine vorzeitliche Vergletscherung des Vulkanmassivs nachweisen (Meyer 1900). Die von ihm dabei identifizierten Gletscher benannte er dabei nach Glazialgeologen oder nach Forschungsreisenden, die mit ihren Studien zum Erkenntnisgewinn über die Beschaffenheit des Kilimandscharo beigetragen hatten (Abb. 3): Credner-Gletscher, Drygalski-Gletscher, Penck-Gletscher, Heim-Gletscher, Decken-Gletscher, Kersten-Gletscher, Rebmann-Gletscher. ${ }^{4}$ Zwischen dem Penck-Gletscher und Heim-Gletscher identifizierte Meyer zwei weitere Gletscher, die er der damaligen Nomenklatur für eine radiale Erosionsrinne in Steilhängen von Vulkanen entsprechend Großer und Kleiner BarrancoGletscher benannte.

In seinem Buch „Der Kilimandscharo“ (Meyer 1900) lieferte er für etliche von ihm initiierte Benennungen Begründungen, insbesondere für die Verwendung von Personennamen. Bei genauer Betrachtung dieses Namensgutes lassen sich Muster und wohl auch Absichten erkennen, die mit seinen Forschungsinteressen im Zusammenhang stehen (Crom 2019). Darüber hinaus hat er aber auch seine Reisegefährten mit der Benennung markanter Landschaftselemente geehrt. Der Bergsteiger Ludwig Purtscheller

\footnotetext{
${ }^{4}$ Hermann Credner (1841-1913), Erich von Drygalski (1865-1949), Albrecht Penck (1858-1945), Albert Heim (1847-1939), Carl Claus von der Decken (1833-1865), Otto Kersten (1839-1900), Johannes Rebmann (1820-1876).
} 


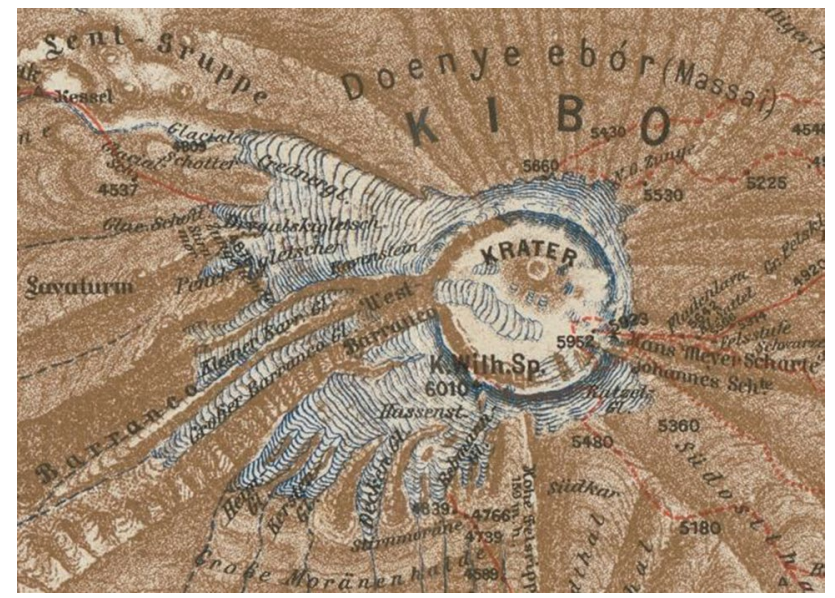

Abb. 3 Ausschnitt aus Meyer/Krauss Spezialkarte des Kilimandjaro 1:100.000. Beilage zu Meyer 1900. Quelle: Staatsbibliothek zu Berlin Us 1109-51a

(1849-1900), der mit ihm 1889 den Gipfel des Kibo er reicht hatte, ist Namensgeber für die zweithöchste Spitze im Bereich des Mawensi, während nach dem Bergmaler Ernst Platz (1867-1940) ein solitärer Vulkankegel nördlich des Schira-Kammes benannt wurde. Zudem hat Hans Meyer andere Wissenschaftler oder Personen der Kolonialverwaltung geehrt, so dass sich in den Karten die Toponyme Bismarck-Hügel, Johannes-Scharte, Krapf-Hügel, Lentgruppe, Liebert-Spitze, Moltke-Stein, Volkens-Hügel oder WissmannSpitze $e^{5}$ finden. Meyer selbst ist mit der Bezeichnung HansMeyer-Scharte eingeschrieben, die einen Einschnitt auf dem Ringwall oberhalb des Ratzel-Gletschers bezeichnet. Die Bildung dieser Toponyme erfolgte durch die Kombination des Personennamens mit einem Appellativ.

\subsection{Ravenstein und Hassenstein}

Zwei Namen aber stechen aus den Schöpfungen Meyers hervor, da sie keine Gattungsbezeichnung erhalten haben, denn diese ist bereits Bestandteil des Personennamens bei Ravenstein und Hassenstein. In beiden Fällen handelte es sich um markante Felsen, die bei ihrer Entdeckung aus der Eisumklammerung der Kibo-Gletscher herausragten, so dass die Verwendung der Namen wegen des Namensbestandteils „Stein“ ideal passte. Sie wurden von Hans Meyer ohne weitere Gattungsbezeichnung verwendet, da der hintere

\footnotetext{
5 Otto von Bismarck (1815-1898), Kurt Johannes (1864-1913), Johann Ludwig Krapf (1810-1881), Carl Lent (1867-1894), Eduard von Liebert (1850-1934), Helmuth von Moltke (1800-1891), Georg Volkens (1855-1917), Hermann von Wissmann (1853-1905).
}

Bestandteil der Namen als solcher gelesen und verstanden werden kann. Der Ravenstein ist im westlichen Gletscherfeld im Bereich des Penck- und Drygalski-Gletschers verortet, während der Hassenstein im südlichen Gletscherfeld im oberen Bereich des Rebmann-Gletschers zu finden ist (Abb. $3)$. Meyer hat in seinen Werken keine Hinweise auf diese Namensgebungen hinterlassen, so dass über ihre Entstehung nur spekuliert werden kann.

Kartographen bekommen eher selten eine Ehrung durch Einschreibungen zugesprochen. Zu diesen zählen beispielsweise Peter und Philipp Apian, Carl Diercke, Tobias Mayer, Gerhard Mercator, Friedrich August Ravenstein oder Daniel Sotzmann, die in ihren Heimat- oder Wirkungsorten durch die Benennung einer Straße einen lokalen Niederschlag gefunden haben. In Gotha findet sich gleich eine größere Zahl an geehrten Kartographen: Hermann Haack, August Petermann, Adolf Stieler oder Carl Vogel sind als Straßennamen ins örtliche Gedächtnis eingeschrieben worden. Nürnberg hat im Ortsteil Langwasser sogar ein regelrechtes Straßencluster mit Johann Gabriel Doppelmayr, Erhard Etzlaub, Reinhard Gensfelder, Johann Baptist Homann und Georg Nöttelein benannt. In Einzelfällen haben auch Gebäude (Schulen) den Namen eines Kartographen erhalten. Gerhard Mercator ist dabei eine Ausnahmeerscheinung, denn ihm wurde nicht nur zum 500. Geburtstag eine Briefmarke gewidmet, sondern er fungiert als Namenspatron sogar extraterrestrisch für einen Mondkrater und einen Asteroiden. Doch als Wortspiel mit einem Namensbestandteil, das einen zuzufügenden Gattungsbegriff ersetzt, bleiben Ravenstein und Hassenstein Sonderfälle.

Der Ravenstein und der Hassenstein sind nicht näher klassifizierte Landschaftselemente, doch dürfte es sich um Lavafelstürme handeln. Sie eigneten sich besonders für Peilungen, da sie aufgrund ihrer exponierten Lage im Gletscherumfeld von verschiedenen Positionen aus sichtbar waren, soweit es die Witterungsbedingungen wohl zuließen. So beschreibt Hans Meyer eine vergleichbare Szene: „Über ihr erhebt sich in wundervollem Linienschwung ein stolzer, über 1000 Fuss hoher Felsturm auf dem Nordostgrat des Mawensi, der mir wegen seiner Gestalt und Lage immer als hervorragender Peilpunkt gedient hat und als solcher auch künftig anderen Kartographen dienen wird" (Meyer 1900, S. 155). Während seiner 1898 durchgeführten Forschungsreise zum Kilimandscharo hatte er am Kibo zwei Vorstöße in die eisigen Höhen bis zu den jeweiligen Gletscherstirnen unternommen, zunächst zum Drygalski-Gletscher und später zum Rebmann-Gletscher, wobei ihm die Lavafelsen als gut sichtbare Zielmarken aufgefallen sein dürften. Die Anregung zum Wortspiel könnte durch Geierraben (Corvus albicollis) hervorgerufen worden sein, die von ihm vereinzelt in 
der großen Höhe angetroffen wurden. Von der englischen Bezeichnung raven ist eine schnelle Verbindung zu Ravenstein herzustellen und so dürfte Meyer möglicherweise Ernst Georg Ravenstein (1834-1913) vor Augen gestanden haben, der 1852 zu August Petermann (1822-1878) nach London ging und zwischen 1855 und 1875 als Kartograph beim britischen Kriegsministerium beschäftigt war. Später hatte er eine viel beachtete Karte vom östlichen Äquatorialafrika erstellt (Bederman 1992). Vielleicht wollte Meyer aber auch die gesamte kartographische Dynastie Ravenstein ehren, die von Friedrich August Ravenstein (1809-1881) 1830 mit dem Aufbau von Ravensteins Geographischer Verlagsanstalt begründet wurde und die über viele Generationen tätig war (Ferschke 1980, Ravenstein 1980). Schließlich arbeiteten der Ravenstein-Verlag und das Verlagshaus Meyer (Bibliographisches Institut), aus dem Hans Meyer stammte (Brogiato 2008), im kartographischen Bereich eng zusammen (Espenhorst 1994, 2003).

Einmal durch das Wortspiel angeregt, war die Übertragung zu Hassenstein für einen aus dem oberen RebmannGletscher herausschauenden Lavafelsen nur noch ein kleiner Schritt. Bruno Hassenstein (1839-1902), ab 1854 ebenfalls von August Petermann nun bei Perthes in Gotha ausgebildet, entwickelte sich dort zu einem der führenden Kartographen (Köhler 2002). Er hatte die bereits oben erwähnten Karten des Kilimandscharo-Gebietes sowie einige weitere Karten für die Publikationen anderer Afrika-Forscher verantwortet. 1890 hatte er schließlich die Karten für Meyers Werk Ostafrikanische Gletscherfahrten „konstruirt und gezeichnet“, wie es im Titel der Karte selbst heißt. In den Karten von Paul Krauss zu Meyers Buch „Der Kilimandscharo“ von 1900 sind nun beide Namen gut lesbar enthalten, allerdings ist die Zuordnung der Beschriftung zu den eingezeichneten Felsen nicht ganz eindeutig.

\subsection{Folgen des Gletscherrückgangs}

Diese erfreuliche Anerkennung der kartographischen Leistungen von Ravenstein und Hassenstein findet ihren Niederschlag ausschließlich in Karten, da die benannten Vulkanfelsen in unzugänglichen Gebieten stehen. Der Ravenstein ist in den von Fritz Jäger 1909 publizierten Karten zum westlichen Kibo namentlich sichtbar (Abb. 4), während der Hassenstein außerhalb des Untersuchungsgebietes lag. Jäger (1881-1966) hatte mehrere Reisen nach Ostafrika unternommen, u. a. mit Carl Uhlig (1872-1938)

\footnotetext{
${ }^{6}$ Weitere kleine Gletscher wurden später, insbesondere durch den fortschreitenden Rückzug der großen Gletscher entdeckt und benannt. Der Arrow-Glacier ist ein Rest des Kleinen Barranco-Gletschers. Die auf einer Seite des Mount-Kilimanjaro-Wiki (http://kilimanjar
}

oder seinem Vetter Eduard Oehler (1881-1941). Fritz Klute (1885-1952) unternahm 1912 ebenfalls in Begleitung von Oehler eine Forschungsreise, um Studien zur Vergletscherung des Kilimandscharo zu betreiben, wobei er die Gletscher erstmals photogrammetrisch vermessen hat (Klute 1920, 1921). Seine Karte im Maßstab 1:50.000 (Abb. 5) wurde für alle nachfolgenden Untersuchungen zur Glaziologie des Gebietes maßgeblich (Cullen et al. 2013). ${ }^{6}$ Sie zeigt und benennt wiederum den Ravenstein, während der Hassenstein namentlich keine Entsprechung findet. Seine Verortung war aufgrund der spärlichen Datengrundlage nach Meyers Arbeiten wohl zu vage. Zwar ist im oberen Bereich des Rebmanngletschers ein Felsen eingezeichnet, aber ob es sich dabei um den Hassenstein handeln könnte, bleibt ungewiss.

Beide Forscher, Jäger und Klute, setzten die von Hans Meyer eingeführte Benennungspraxis fort. So finden sich in ihren Karten nun der Uhlig-Gletscher, der Hans-Meyer-Grat, der Lentgrat als östliche Fortsetzung der Lentgruppe oder der Kleine Penck-Gletscher als eigenständiger Gletscher, den Klute zur besseren Unterscheidung nun vom Großen Penck-Gletscher abgrenzt. Mit den Toponymen Oehlergrat und Oehlertal wird zudem die Ehrbekundung des Reisebegleiters fortgesetzt. Die bei Jäger und Klute neu anzutreffenden Landschaftsnamen sind eine Folge der genaueren Vermessung und Analyse der glazialen und vulkanischen Erscheinungen, aber auch der sich verändernden Landschaft. Dadurch ist aber dem Ravenstein und dem Hassenstein keine bleibende Erinnerung beschieden, denn der bereits von Hans Meyer 1900 postulierte und seitdem beschleunigte Gletscherrückgang hat beiden ihre herausragende Stellung gekostet. Waren sie ursprünglich aus der Eisumklammerung als weithin sichtbare Landmarken erkennbar gewesen, so sind sie mit der fortschreitenden Schmelze der Eismassen im Umfeld der seitdem zutage getretenen Vulkangesteine nicht mehr als solche wahrnehmbar und inzwischen aus den aktuellen Karten verschwunden. Lediglich eine Trekkingkarte aus dem Jahr 2012 trägt in einer Beikarte des Kibo im Maßstab 1:50.000 noch das Toponym Ravenstein ohne örtlichen Bezugspunkt (Szyczak 2012). So haben sich ihre Spuren bereits verloren, ein Schicksal, dem die Namensgeber der großen Gletscher am Kilimandscharo folgen werden.

\footnotetext{
Footnote 6 (continued)

o.bplaced.net/wiki/index.php?title=Irrtümer_zum_Kilimanjaro\#Der_ von_Pfeil_Gletscher) zu findende Interpretation ist irrig, einen PfeilGletscher als ursprüngliche Bezeichnung des Arrow-Glacier in Erinnerung an den Kolonialmachthaber Joachim von Pfeil (1857-1924) hat es nie gegeben. Er ist in keiner Karte benannt und hätte auch nicht in das von Hans Meyer geprägte Benennungsschema gepasst.
} 


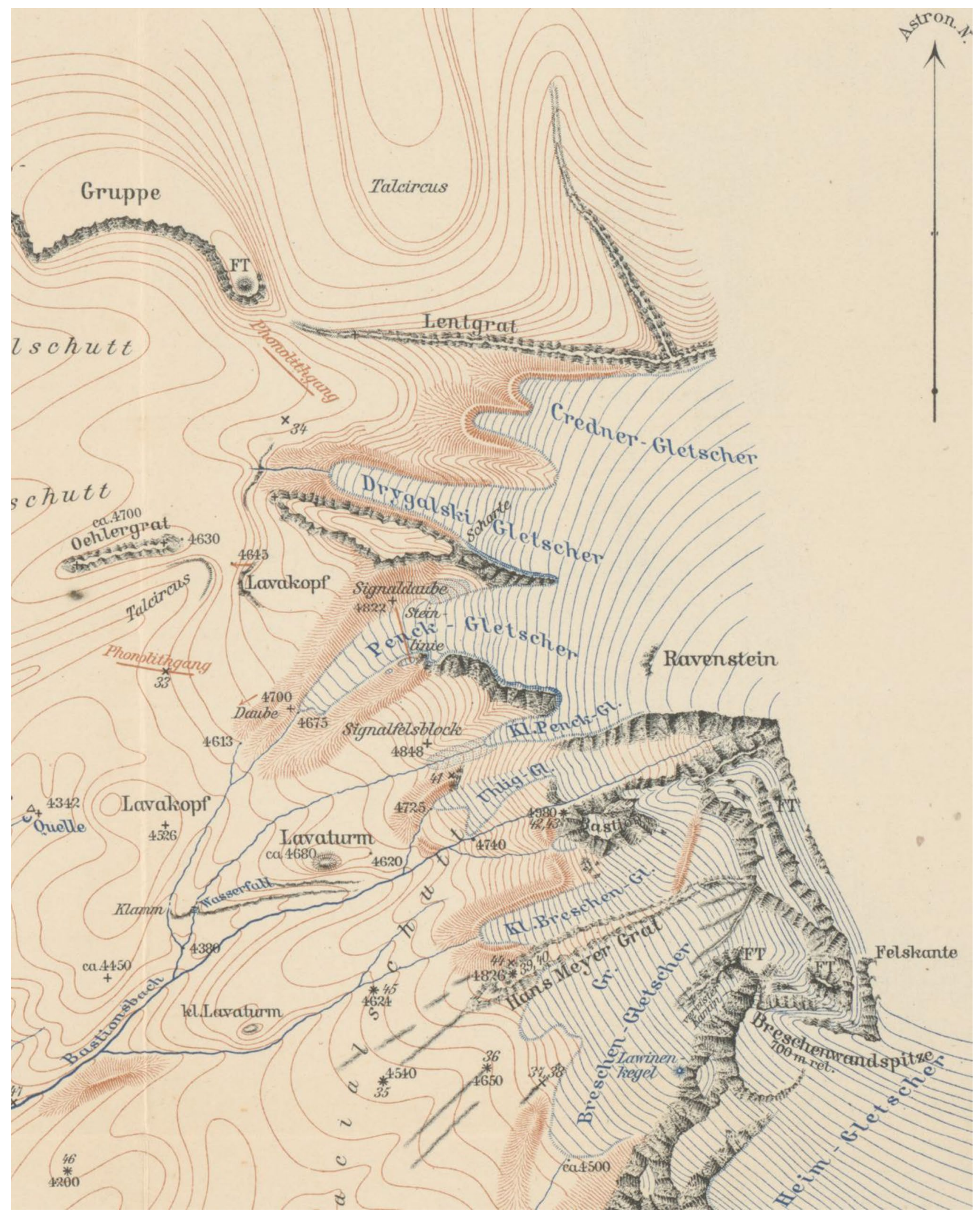

Abb. 4 Ausschnitt aus Jäger Westlicher Kibo 1:40.000. Beilage zu Jäger 1909. Quelle: Staatsbibliothek zu Berlin Kart. C 16,960-90 


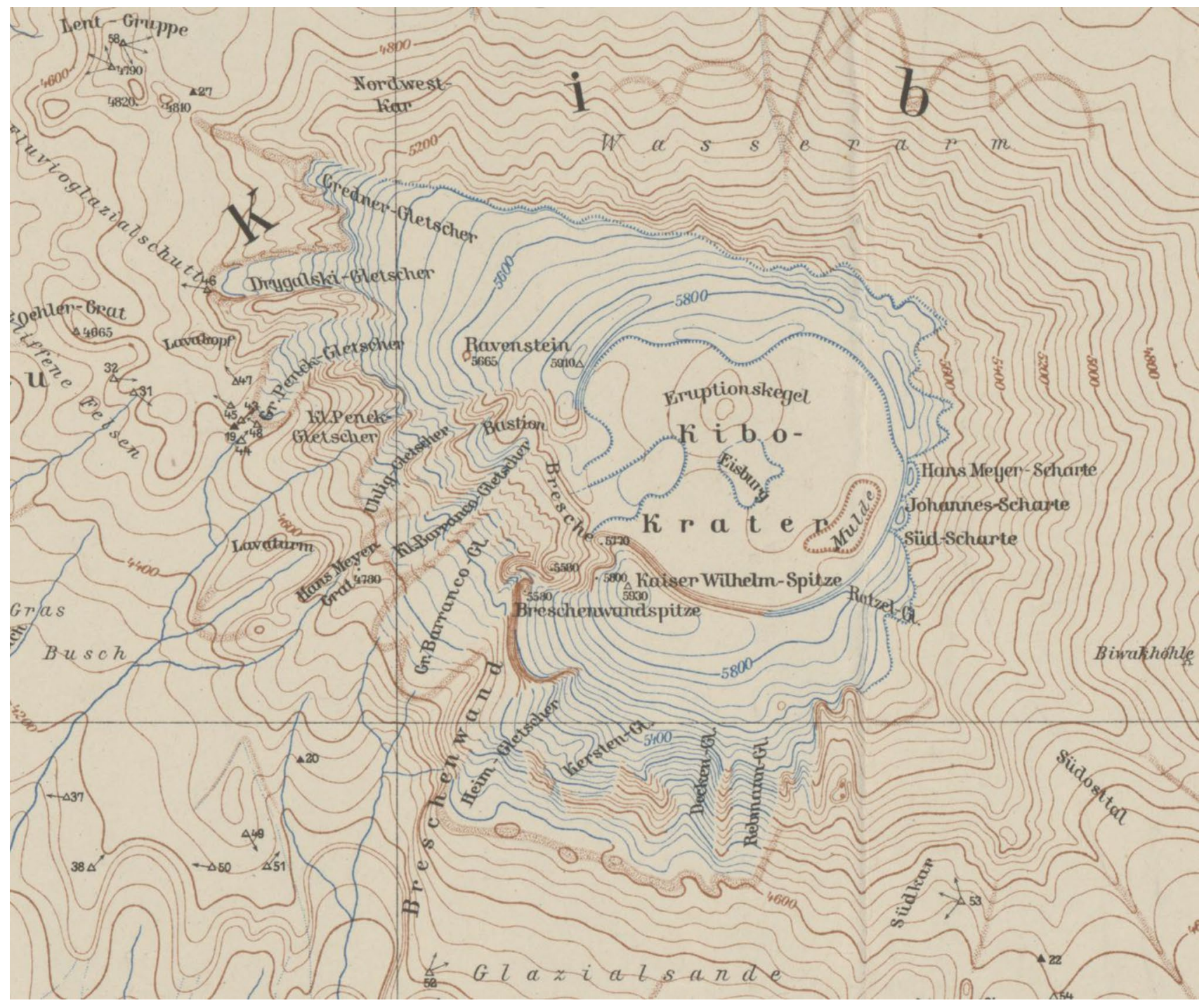

Abb. 5 Ausschnitt aus Klute Kilimandscharo 1:50.000. Beilage zu Klute 1920 und 1921. Quelle: Staatsbibliothek zu Berlin Kart. GfE 5, 479

Funding Open Access funding enabled and organized by Projekt DEAL.

Open Access This article is licensed under a Creative Commons Attribution 4.0 International License, which permits use, sharing, adaptation, distribution and reproduction in any medium or format, as long as you give appropriate credit to the original author(s) and the source, provide a link to the Creative Commons licence, and indicate if changes were made. The images or other third party material in this article are included in the article's Creative Commons licence, unless indicated otherwise in a credit line to the material. If material is not included in the article's Creative Commons licence and your intended use is not permitted by statutory regulation or exceeds the permitted use, you will need to obtain permission directly from the copyright holder. To view a copy of this licence, visit http://creativecommons.org/licenses/by/4.0/.

\section{Literatur}

Bederman SH (1992) The Royal Geographical Society, E. G. Ravenstein, and a map of Eastern Equatorial Arfica-1877-1883. Imago Mundi 44:106-119. https://doi.org/10.1080/03085699208592744

Brogiato HP (2008) Meyers Universum: Zum 150. Geburtstag des LeipzigerVerlegers und Geographen Hans Meyer (1858-1929). Leibniz-InstitutfürLänderkunde, Leipzig

Brunner K (1989) Erstbesteigung und erste Karten des Kilimandscharo. KartographischeNachrichten 39:216-222

Brunner K (2004) Frühe Karten des Kilimandscharo: Ein Beitrag zur Expeditionskartographie. Cartographica Helvetica 30:3-9

Crom W (2019) German Names in the Kilimanjaro Region. In: Kent A et al (eds) Mapping empires: colonial cartographies of land and sea. 7th international symposium of the ICA commission on 
the history of cartography (=Lecture Notes in Geoinformation and Cartography), 2018. Springer, Cham, S. 229-246

Cullen NJ, Sirguey P, Mölg T, Kaser G, Winkler M, Fitzsimons SJ (2013) A century of ice retreat on Kilimanjaro: the mapping reloaded. Cryosphere 7:419-431

Demhardt IJ (2004) Die Kartographie des Kaiserlichen Schutzgebietes Deutsch-Ostafrika. Cartographica Helvetica 30:11-21

Espenhorst J (2003) Petermann's planet: a guide to German handatlases and their siblings throughout the world 1800-1950, volume 1: The great hand-atlases. Pangaea, Schwerte

Espenhorst J (1994) Andree, Stieler, Meyer \& Co: bibliographisches Handbuch. Handatlanten des deutschen Sprachraumes. Pangaea, Schwerte, pp 1800-2194

Ferschke H (1980) 150 Jahre Ravenstein-Landkarten. Kartographische Nachrichten 30:229-232

Hassenstein B (1864) Das Gebiet der Schneeberge Kilima-Ndscharo und Kenia in Ostafrika. Nach den Reiseberichten der Missionäre Krapf \& Rebmann, 1844-53, und auf Grund der Forschungen K. van der Decken's, 1861 u. 1862, von Burton, Speke (1857), Owen (1824) u. A. 1:1.500.000. Petermanns Geographische Mitteilungen [10]: Tafel 16. https://zs.thulb.uni-jena.de/receive/jport al_jparticle_00512878

Hassenstein B (1868) Das Gebiet der beidenReisen des Baron C. C. von der DeckenzumSchneebergKilima-Ndscharo in den Jahren 1861 and 1862. 1:1.000.000. Kraatz, Berlin

Hettner A (1927) Die Geographie: Ihre Geschichte, ihrWesen und ihreMethoden. Hirt, Breslau

Jäger F (1909) Forschungen in den Hochregionen des Kilimandscharo. Mitteilungenaus den deutschenSchutzgebieten 22:113-197

Kiepert H (1863) Karte des Schneegebirges Kilima-Ndjaro aufgenommen von Baron C. von der Decken auf seiner ersten Reise. 1:500.000. Zeitschrift für allgemeine Erdkunde NF XV, Tafel V. http://www.digizeitschriften.de/download/PPN3913656 22_1863_0015/PPN391365622_1863_0015__log65.pdf

Klute F (1921) Die stereogrammetrische Aufnahme der Hochregion des Kilimandscharo. Zeitschrift der Gesellschaft für Erdkunde zu Berlin 56:144-151

Klute F (1920) Ergebnisse der Forschungen am Kilimandscharo 1912. Reimer, Berlin
Köhler F (2002) Gotha galt als Mekka der Kartographen: Vor 100 Jahren verstarb Bruno Hassenstein. Zwischen Fahner Höh ' und Rennsteig 12: 18

Kolonialabtheilung des Auswärtigen Amtes (eds) (1901-1914) Großer deutscher Kolonialatlas. Reimer, Berlin

Meyer H (1890) Ostafrikanische Gletscherfahrten. Forschungsreisen im Kilimandscharo-Gebiet. Duncker \& Humblot, Leipzig

Meyer H (1900) Der Kilimandscharo: Reisen und Studien. Reimer, Berlin

Petermann A (1859) Originalkarte von Burton's u. Speke's Entdeckungen in Inner-Afrika 1857 u. 1858. Nebst Angaben aller übrigen im Bereich der Karte von Europäischen Reisenden zurückgelegten Routen. Mit Benutzung einer Originalzeichnung von Capt. J. H. Speke. 1:7.000.000. Petermanns Geographische Mitteilungen 5. Taf. 15. https://zs.thulb.uni-jena.de/rsc/viewer/jportal_deriv ate_00260681/ThULB_129489816_1859_Perthes_0421.tif

Pillewizer W (1941) Der Anteil der Geographie an der kartographischen Erschließung Deutsch-Ostafrikas. Jahrbuch der Kartographie [1]: 45-175.

Ravenstein H (1980) Die Chronik des VerlagshausesRavenstein : Eine Geschichte ausdemZeitalter der Pioniere, der Entdecker der Erfinder und der Verlagsgründer. Ravenstein, Frankfurt am Main

Rebmann J (1850) Imperfect Sketch of a Map from $1 \frac{1 / 2^{\circ}}{}$ North, to 10 $1 / 2^{\circ}$ South Latitude, and from 29 to 44 degrees East Longitude. Rabbai Mpia: by the Missionaries of the Church Mission ${ }^{y}$ Society in Eastern Africa.

Sprigade P, Moisel M (1914) Die Aufnahmemethoden in den deutschen Schutzgebieten und die deutsche Kolonial-Kartographie. Zeitschrift der Gesellschaft für Erdkunde zu Berlin [49], S. 527-545 http://www.digizeitschriften.de/dms/img/?PID=PPN39 1365657_1914\%7CLOG_0181

Stolz T, Warnke IH (2015) Aspekte der kolonialen und postkolonialen Toponymie unter besonderer Berücksichtigung des deutschen Kolonialismus. In: Schmidt-Brücken D et al (eds) Koloniallinguistik-Sprache in kolonialen Kontexten (=Koloniale und Postkoloniale Lingistik, 8). DeGruyter, Berlin, pp 107-176

Szyczak M (2012) Africa-the hightest peaks: Kilimanjaro, Mount Kenya. Rwenzori, Warszawa 